\title{
Hybrid Analog-Digital Transceiver Designs for Cognitive Radio Millimiter Wave Systems
}

\author{
Christos G. Tsinos, Sina Maleki, Symeon Chatzinotas and Björn Ottersten \\ SnT-Interdisciplinary Centre for Security, Reliability and Trust, University of Luxembourg, \\ Emails: \{christos.tsinos,sina.maleki,symeon.chatzinotas,bjorn.ottersten\}@uni.lu
}

\begin{abstract}
Recent advances in Milimeter wave (mmWave) band mobile communications may provide solutions to the increasing traffic demand in modern wireless systems. Even though mmWave bands are scarcely occupied, the design of a prospect transceiver should guarantee the efficient coexistence with the incumbent services in these bands. To that end, in this paper, two underlay cognitive transceiver designs are proposed based on a hybrid Analog/Digital transceiver architecture that enables the mmWave spectrum access while controlling the interference to the incumbent users with low hardware complexity and power consumption. The first cognitive solution designs a codebook free cognitive hybrid pre-coder by maximizing the mutual information between its two ends subject to interference, power and hardware constraints related to the analog counterpart. The second solution is codebook based and exhibits less complexity than the first one at the cost of inferior spectral efficiency. A novel codebook free solution for the post-coder at the cognitive receiver part is further proposed, based on a hardware constrained Minimum Mean Square Error criterion. Simulations study the performance of both the proposed hybrid approaches and compare it to the one of a fully digital solution for typical wireless environments.
\end{abstract}

\section{INTRODUCTION}

Exponentially increasing demand for higher data rates as well as the spectrum congestion in the lower parts of the electromagnetic spectrum forces the exploration of systems for frequencies within the so-called mmWave band [1]. However, the development of communications systems for mmWave bands is a challenging task. MmWave signals suffer from severe propagation loss, penetration loss and rain fading compared to signals in lower frequencies [2]. Fortunately, the short wavelength of mmWave frequencies enables the denser packing of more antennas in the transceiver, enabling large array structures for providing high beamforming gains and/or improving the system's spectral efficiency.

The conventional fully digital pre/post-coding techniques, developed for lower frequency MIMO systems, are independent of the carrier frequency, though they cannot be applied in mmWave ones due to the one RF chain per antenna requirement that results in high demands in hardware complexity and power consumption. Thus, a digital only mmWave transceiver seems to be currently impractical and to that end, hybrid Analog/Digital (A/D) architectures are examined in literature.

A hybrid approach is based on a two stage setup that consists of a low dimensional digital pre-coder applied in the BaseBand (BB) and an analog beamformer applied in the $\mathrm{RF}$ domain [3]. The RF processing part is implemented via a network of variable phase shifters. A number of different hybrid approaches [3]-[5], was developed recently.

In existing mmWave approaches, the wireless system is assumed as a stand-alone MIMO communications system. Considering the fact that mmWave bands are also allocated to several services (e.g. point-to-point (P2P) or point-tomultipoint (P2MP) backhaul microwave links, satellite links, high resolution radar, radio astronomy, amateur radio e.t.c.) [6], such an assumption seems to be very optimistic. Therefore, it is necessary to take constraints on the imposed interference on the incumbent services into account while designing the mmWave transceivers. This is the subject of this paper.

In detail, the contributions of the present paper are as follows. Two cognitive hybrid analog/digital transceiver designs for point-to-point MIMO systems are presented with the view to maximize the spectral efficiency subject to interference to the Primary User (PU), power and hardware related (limited number of RF chains) constraints. The first approach is a codebook free one and the second a codebook based one.Both of the proposed approaches are based on the underlay cognitive radio paradigm [7] where the Secondary User (SU) may access a spectrum area licensed to a PU, simultaneously with the latter and provided that the interference power on the PU transmissions is below a predefined threshold. The proposed approaches derive the pre-coding and post-coding (combining) matrices by decoupling the transmitter receiver optimization problem. Theoretical results regarding the convergence of the first approach are presented and the performance of both of the approaches is evaluated via simulations and compared to the one of the corresponding fully digital solution.

The remainder of the paper is organized as follows. In Section II, the system description is given. in Section III, the description of the optimal digital only solutions is given. Section IV describes the codebook free hybrid transceiver design. In Section V, the codebook based hybrid transceiver design is developed and Section VI presents some numerical results and the related discussion.

\section{SYSTEM MODEL}

Let us assume that a $\mathrm{SU} R_{s} \times T_{s}$ MIMO system access a spectrum area licensed to a $R_{p} \times T_{p}$ PU under a typical cognitive underlay approach (Fig. 1) [7]. The SU system (Fig. 1) is assumed to be equipped with $N_{s t}<<T_{s}$ and $N_{s r}<<R_{s}$ $\mathrm{RF}$ chains at the transmitter and the receiver, respectively and transmits a number of $L_{s} \leq \min \left\{N_{s t}, N_{s r}\right\}$ streams. Furthermore, the SU system applies a $T_{s} \times L_{s}$ hybrid precoding matrix at the transmitter given by $\mathbf{F}=\mathbf{F}_{R F} \mathbf{F}_{B B}$ where $\mathbf{F}_{R F}$ is the $T_{s} \times N_{s t}$ analog RF precoder implemented via a network of phase shifters and $\mathbf{F}_{B B}$ is the $N_{s t} \times L_{s}$ digital $\mathrm{BB}$ one. In a similar manner, the $R_{s} \times L_{s}$ post-coding matrix is given by $\mathbf{W}=\mathbf{W}_{R F} \mathbf{W}_{B B}$ where $\mathbf{W}_{R F}$ is the $R_{s} \times N_{s r}$ analog post-coding matrix and $\mathbf{W}_{B B}$ is the $N_{s r} \times L_{s}$ digital $\mathrm{BB}$ one, respectively.

The received signal $y_{s}$ at the SU receiver for a narrow-band block fading propagation channel is given by

$$
\mathbf{y}_{s}^{\prime}=\mathbf{W}^{H} \mathbf{y}_{s}=\mathbf{W}^{H}\left(\mathbf{H}_{s s} \mathbf{F} \mathbf{x}_{s}+\tilde{\mathbf{H}}_{s p} \mathbf{x}_{p}+\mathbf{n}_{s}\right)
$$

where $\mathbf{H}_{s s}$ is the $R_{s} \times T_{s}$ channel matrix between the two SU 


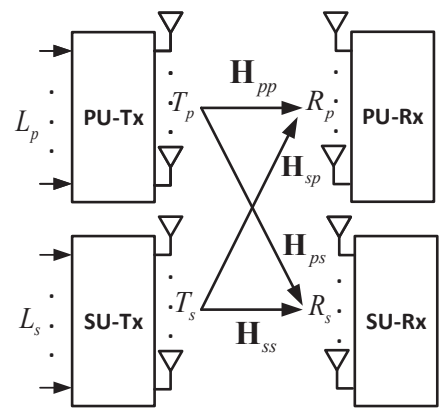

Fig. 1. PU-SU transceiver pairs. The SU is equipped with $N_{s t}<<T_{s}$ and $N_{s r}<<$ $R_{s}$ RF chains at the transmitter's and receiver's side, respectively.

ends, $\mathbf{x}_{s}$ is the $L_{s} \times 1$ vector of the SU transmitted symbols such that $\mathbb{E}\left\{\mathbf{x}_{s} \mathbf{x}_{s}^{H}\right\}=\sigma_{s}^{2} \mathbf{I}_{T_{s}}, \mathbb{E}\{\cdot\}$ is the expectation operator, $\sigma_{s}^{2}$ is the variance of the symbols transmitted by the SU transmitter, $\mathbf{I}_{T_{s}}$ is the $T_{s} \times T_{s}$ identity matrix, $\tilde{\mathbf{H}}_{s p}=\mathbf{H}_{s p} \mathbf{F}_{p}$, $\mathbf{H}_{s p}$ is the $R_{s} \times T_{p}$ channel matrix between the PU transmitter and the $\mathrm{SU}$ receiver and $\mathbf{F}_{p}$ is the $T_{p} \times L_{p}$ pre-coding matrix applied at the PU transmitter, $L_{p} \times 1$ is the number of the transmitted PU streams, $\mathbf{x}_{p}$ is the $L_{p} \times 1$ vector of the PU transmitted symbols such that $\mathbb{E}\left\{\mathbf{x}_{p} \mathbf{x}_{p}^{H}\right\}=\sigma_{p}^{2} \mathbf{I}_{T_{p}}, \sigma_{p}^{2}$ is the variance of the symbols transmitted by the PU transmitter and $\mathbf{n}_{s}$ is i.i.d. complex Gaussian noise modeled as $\mathcal{C N}\left(0, \sigma_{n}^{2} \mathbf{I}_{R_{s}}\right)$.

The received signal at the PU receiver is given by

$$
\mathbf{y}_{p}=\mathbf{H}_{p p} \mathbf{F}_{p} \mathbf{x}_{p}+\mathbf{H}_{p s} \mathbf{F} \mathbf{x}_{s}+\mathbf{n}_{p},
$$

where $\mathbf{H}_{p p}$ is the $R_{p} \times T_{p}$ channel matrix between the two $\mathrm{PU}$ ends, $\mathbf{H}_{p s}$ is the $R_{p} \times T_{s}$ channel matrix between the SU transmitter and the PU receiver and $\mathbf{n}_{p}$ is i.i.d. complex Gaussian noise modeled as $\mathcal{C N}\left(0, \sigma_{n}^{2} \mathbf{I}_{R_{p}}\right)$. A geometrical channel model is assumed for all the involved channels that captures better the characteristics of the mmWave band which is described in detailed in [3], [5].

Let us now assume that the SU has perfect knowledge of $\mathbf{H}_{s s}, \tilde{\mathbf{H}}_{s p}$ and $\mathbf{H}_{p s}$ channels. Note that this is a typical requirement in $\mathrm{CR}$ underlay approaches [7]. The aim is to derive the pre/post-coding matrices in order to maximize the spectral efficiency of the SU system subject to constraints on the total transmission power $\left(P_{\max }\right)$ and on the interference to the PU transmissions $\left(I_{\max }\right)$. To that end, the following optimization problem is defined.

$$
\begin{aligned}
\left(\mathcal{P}_{1}\right): & \max _{\mathbf{F}, \mathbf{W}} R(\mathbf{F}, \mathbf{W}) \\
& \text { s.t. } \operatorname{tr}\left(\mathbf{F} \mathbf{F}^{H}\right) \leq P_{\max } \& \operatorname{tr}\left(\mathbf{H}_{p s} \mathbf{F} \mathbf{F}^{H} \mathbf{H}_{p s}^{H}\right) \leq I_{\max }
\end{aligned}
$$

where the spectral efficiency $R(\mathbf{F}, \mathbf{W})$ is defined as

$$
R(\mathbf{F}, \mathbf{W})=\log _{2} \operatorname{det}\left(\mathbf{I}_{L_{s}}+\mathbf{R}_{n}^{-1} \mathbf{W}^{H} \mathbf{H}_{s s} \mathbf{F} \mathbf{F}^{H} \mathbf{H}_{s s}^{H} \mathbf{W}\right),
$$

with $\mathbf{R}_{n}=\mathbf{W}^{H}\left(\tilde{\mathbf{H}}_{s p} \tilde{\mathbf{H}}_{s p}^{H}+\sigma_{n}^{2} \mathbf{I}_{L_{s}}\right) \mathbf{W}$ denoting the interference plus noise covariance matrix at the SU receiver and $\operatorname{det}(\cdot)$ is the determinant of a matrix. For the sake of simplicity, we consider in this paper the case where interference constraints are posed from only one PU. Extensions to the multiple PU case are straightforward by adding the corresponding interference related constraints on $\left(\mathcal{P}_{1}\right)$.

\section{Digital Only Solution}

In the digital only design, the SU pre/post-coding matrices can be found by solving directly $\left(\mathcal{P}_{1}\right)$ under the assumption that both the transceiver ends have equal number of RF chains and antennas. This problem is in general intractable [8] and the common approach is to temporally decouple the designs at the transmitter and the receiver. To that end, the optimal precoding matrix $\mathbf{F}_{D}$ is designed such that the mutual information achieved by Gaussian signaling over the wireless channel is maximized. Thus, the following optimization problem should be solved

$$
\begin{aligned}
& \left(\mathcal{P}_{2}\right): \max _{\tilde{\mathbf{F}}} \log _{2} \operatorname{det}\left(\mathbf{I}_{L_{s}}+\mathbf{Q}^{-1 / 2} \mathbf{H}_{s s} \tilde{\mathbf{F}} \mathbf{H}_{s s}^{H} \mathbf{Q}^{-1 / 2}\right) \\
& \text { s.t. } \operatorname{tr}(\tilde{\mathbf{F}}) \leq P_{\max } \& \operatorname{tr}\left(\mathbf{H}_{p s} \tilde{\mathbf{F}} \mathbf{H}_{p s}^{H}\right) \leq I_{\max } \& \tilde{\mathbf{F}} \succeq \mathbf{0}
\end{aligned}
$$

where $\mathbf{A} \succeq \mathbf{0}$ denotes a positive semi-definite matrix, $\mathbf{Q}=$ $\tilde{\mathbf{H}}_{s p} \tilde{\mathbf{H}}_{s p}^{H}+\sigma_{n}^{2} \mathbf{I}_{R_{s}}$ is the covariance matrix of the interference plus noise signal and $\tilde{\mathbf{F}}=\mathbf{F}_{D} \mathbf{F}_{D}^{H}$ [9]. Note that $\left(\mathcal{P}_{2}\right)$ is convex and thus, it can be solved efficiently by standard convex optimization techniques (i.e. interior point methods [10]). Then, by the Eigenvalue Value Decomposition on the optimal matrix $\tilde{\mathbf{F}}^{*}=\mathbf{U}_{F} \boldsymbol{\Sigma}_{F} \mathbf{U}_{F}$, we may find $\mathbf{F}_{D}=\mathbf{U}_{F} \sqrt{\boldsymbol{\Sigma}_{F}}$.

For the receiver's side, a Minimum Mean Square Error (MMSE) approach is considered. That is, matrix $\mathbf{W}$ is derived as the solution to the optimization problem

$$
\min _{\mathbf{W}} \mathbb{E}\left\{\left\|\mathbf{x}_{s}-\mathbf{W}^{H} \mathbf{y}_{s}\right\|_{F}^{2}\right\},
$$

where $\|\cdot\|_{F}$ is the Frobenius norm. The solution is given by

$$
\begin{aligned}
& \mathbf{W}_{M M S E}=\mathbb{E}\left\{\mathbf{y}_{s} \mathbf{y}_{s}^{H}\right\}^{-1} \mathbb{E}\left\{\mathbf{x}_{s} \mathbf{y}_{s}^{H}\right\}^{H} \\
& =\left(\mathbf{H}_{s s} \mathbf{F}_{D} \mathbf{F}_{D}^{H} \mathbf{H}_{s s}^{H}+\tilde{\mathbf{H}}_{s p} \tilde{\mathbf{H}}_{s p}^{H}+\sigma_{n}^{2} \mathbf{I}_{R_{s}}\right)^{-1} \mathbf{H}_{s s} \mathbf{F}_{D}
\end{aligned}
$$

where we used (1)-(2) and the assumption that $\mathbb{E}\left\{\mathbf{x}_{s} \mathbf{x}_{p}^{H}\right\}=$ $\mathbf{0}_{L_{s} \times L_{p}}$ [11], where $\mathbf{0}_{L_{s} \times L_{p}}$ is a $L_{s} \times L_{p}$ matrix of zero entries.

\section{Codebook Free Hybrid Transceiver Design}

In the case of the hybrid approaches, the solution of $\left(\mathcal{P}_{1}\right)$ is even harder to be derived since the constraints related to the phase shifting network force the analog counterparts to lie in the non-convex space of unit modulus complex matrices. In order to achieve a simple solution, the transceiver design should be again temporally decoupled.

Let us first present the pre-coder design by casting $\left(\mathcal{P}_{2}\right)$ into the hybrid pre-coding case, that is

$$
\begin{aligned}
\left(\mathcal{P}_{3}\right): & \max _{\mathbf{F}_{R F}, \mathbf{F}_{B B}} \log _{2} \operatorname{det}\left(\mathbf{I}_{L_{s}}+\tilde{\mathbf{H}}_{s s} \mathbf{F}_{R F} \mathbf{F}_{B B} \mathbf{F}_{B B}^{H} \mathbf{F}_{R F}^{H} \tilde{\mathbf{H}}_{s s}^{H}\right) \\
& \text { s.t. } \mathbf{F}_{R F} \mathbf{F}_{B B} \in \mathcal{S} \& \mathbf{F}_{R F} \in \mathcal{F}
\end{aligned}
$$

where $\mathcal{S}=\left\{\mathbf{A} \in \mathbb{C}^{T_{s} \times N_{s t}} \mid\|\mathbf{A}\|_{F}^{2} \leq P_{\max } \&\left\|\mathbf{H}_{p s} \mathbf{A}\right\|_{F}^{2}\right.$ $\left.\leq I_{\max }\right\}$ and $\mathcal{F}=\left\{\mathbf{A} \in \mathbb{C}^{M \times N}|| \mathbf{A}(m, n) \mid=1,1 \leq m \leq\right.$ $\bar{M}, 1 \leq n \leq N\}$ for an arbitrary matrix $\mathbf{A}$ with complex entries $\mathbf{A}(m, n)$ and $\tilde{\mathbf{H}}_{s s}=\sigma_{s} \mathbf{Q}^{-1 / 2} \mathbf{H}_{s s}$. Problem $\left(\mathcal{P}_{3}\right)$ is non-convex due to the bi-convex cost function and the nonconvex constraint set $\mathcal{F}$. In the following, an efficient solution is derived by employing the so-called ADMM [12]. Let us first write $\left(\mathcal{P}_{3}\right)$ in the following equivalent form

$$
\begin{gathered}
\left(\mathcal{P}_{4}\right): \min _{\mathbf{Z}, \mathbf{F}_{R F}, \mathbf{F}_{B B}}-\log _{2} \operatorname{det}\left(\mathbf{I}_{L_{s}}+\tilde{\mathbf{H}}_{s s} \mathbf{Z} \mathbf{Z}^{H} \tilde{\mathbf{H}}_{s s}^{H}\right)+\mathbb{1}_{\mathcal{S}}\{\mathbf{Z}\}+ \\
\quad \text { s.t. } \quad \mathbf{Z}=\mathbf{F}_{R F}\left\{\mathbf{F}_{R F}\right\} \\
\quad \mathbf{F}_{B B}
\end{gathered}
$$


where $\mathbf{Z}$ is an auxiliary $T_{s} \times N_{s t}$ matrix variable and the indicator function of sets $\mathcal{S}, \mathcal{F}$ is defined as

$$
\mathbb{1}_{\mathcal{S}(\mathcal{F})}\{\mathbf{A}\}=\left\{\begin{array}{l}
\mathbf{A}, \mathbf{A} \in \mathcal{S}(\mathcal{F}) \\
\infty, \quad \mathbf{A} \notin \mathcal{S}(\mathcal{F})
\end{array} .\right.
$$

The augmented Lagrangian function of $\left(\mathcal{P}_{4}\right)$ is given by

$$
\begin{aligned}
& \mathcal{L}_{T}\left(\mathbf{Z}, \mathbf{F}_{R F}, \mathbf{F}_{B B}, \boldsymbol{\Lambda}\right)=-\log _{2}\left(\mathbf{I}_{L_{s}}+\tilde{\mathbf{H}}_{s s} \mathbf{Z} \mathbf{Z}^{H} \tilde{\mathbf{H}}_{s s}^{H}\right)+ \\
& \mathbb{1}_{\mathcal{S}}\{\mathbf{Z}\}+\mathbb{1}_{\mathcal{F}}\left\{\mathbf{F}_{R F}\right\}+\left\langle\boldsymbol{\Lambda}, \mathbf{Z}-\mathbf{F}_{R F} \mathbf{F}_{B B}\right\rangle+ \\
& \frac{\alpha}{2}\left\|\mathbf{Z}-\mathbf{F}_{R F} \mathbf{F}_{B B}\right\|_{F}^{2},
\end{aligned}
$$

where $\langle\mathbf{A}, \mathbf{B}\rangle=\sum_{m, n} \mathbf{A}(m, n) \mathbf{B}(m, n)$, for two matrices $\mathbf{A}$ and $\mathbf{B}, \boldsymbol{\Lambda}$ is the $T_{s} \times N_{s t}$ Lagrange Multiplier matrix and $\alpha$ is a scalar penalty parameter.

Following the ADMM approach, the solution to $\left(\mathcal{P}_{4}\right)$ is given by the following alternating minimization steps $\left(\mathcal{P}_{4 A}\right)$ $\left(\mathcal{P}_{4 D}\right)$

$$
\begin{aligned}
& \mathbf{Z}_{n}=\arg \min _{\mathbf{Z}} \mathcal{L}_{T}\left(\mathbf{Z}, \mathbf{F}_{R F(n-1)}, \mathbf{F}_{B B(n-1)}, \boldsymbol{\Lambda}_{n-1}\right) \\
& \mathbf{F}_{R F(n)}=\arg \min _{\mathbf{F}_{R F}} \mathcal{L}_{T}\left(\mathbf{Z}_{n}, \mathbf{F}_{R F}, \mathbf{F}_{B B(n-1)}, \boldsymbol{\Lambda}_{n-1}\right) \\
& \mathbf{F}_{B B(n)}=\arg \min _{\mathbf{F}_{B B}} \mathcal{L}_{T}\left(\mathbf{Z}_{n}, \mathbf{F}_{R F(n)}, \mathbf{F}_{B B}, \boldsymbol{\Lambda}_{n-1}\right) \\
& \boldsymbol{\Lambda}_{n}=\arg \min _{\boldsymbol{\Lambda}} \mathcal{L}_{T}\left(\mathbf{Z}_{n}, \mathbf{F}_{R F(n)}, \mathbf{F}_{B B(n)}, \boldsymbol{\Lambda}\right)
\end{aligned}
$$

where $n$ is the iteration index. Let us first derive the solution of $\left(\mathcal{P}_{4 A}\right)$. This is a convex problem (with respect to the optimizing matrix parameter $\mathbf{Z}$ ) and its solution will be based on a projected gradient based approach. Thus, $\mathbf{Z}_{n}$ is derived by updating at each step the solution $\mathbf{Z}_{i, n}$ based on the previous one $\mathbf{Z}_{i-1, n}$ as,

$$
\begin{aligned}
\mathbf{Z}_{i, n} & =\Pi_{\mathcal{S}}\left\{\mathbf{Z}_{i-1, n}-\right. \\
& \left.\mu \nabla_{\mathbf{Z}} \mathcal{L}_{T}\left(\mathbf{Z}_{i-1, n}, \mathbf{F}_{R F(n-1)}, \mathbf{F}_{B B(n-1)}, \boldsymbol{\Lambda}_{n-1}\right)\right\},
\end{aligned}
$$

where $\mu$ is a step size parameter, $\nabla_{\mathbf{Z}}$ is the gradient operator with respect parameter $\mathbf{Z}$ and $\Pi_{\mathcal{S}}$ is the projection onto the set $\mathcal{S}$ operator that it can be shown to be given for an arbitrary matrix A [13] by

$$
\Pi_{\mathcal{S}}\{\mathbf{A}\}= \begin{cases}\mathbf{A}, & \mathbf{S} \in \mathcal{S} \\ \mathbf{B}^{-1} \mathbf{A}, & \mathbf{S} \notin \mathcal{S}\end{cases}
$$

where, $\mathbf{B}=\left(1+\lambda_{1}\right) \mathbf{I}_{L_{s}}+\lambda_{2} \mathbf{H}_{p s}^{H} \mathbf{H}_{p s}$ and $\lambda_{1}$ and $\lambda_{2}$ are set such that the power $\left(P_{\max }\right)$ and interference constraints $\left(I_{\max }\right)$ are met, i.e. via a bisection method [14]. The iterations of (15) are running until the following termination criterion is met [13]

Let us move now to the derivation of the solution to $\left(\mathcal{P}_{4 B}\right)$ which can be given by solving the corresponding unconstrained problem and then projecting onto the set $\mathcal{F}$. That is,

$$
\begin{aligned}
\tilde{\mathbf{F}}_{R F(n)} & =\frac{1}{\alpha}\left(\boldsymbol{\Lambda}_{n-1}+\alpha \mathbf{Z}_{n}\right) \mathbf{F}_{B B(n)}^{H}\left(\mathbf{F}_{B B(n)} \mathbf{F}_{B B(n)}^{H}\right)^{-1} \\
\mathbf{F}_{R F(n)} & =\Pi_{\mathcal{F}}\left\{\tilde{\mathbf{F}}_{R F(n)}\right\}
\end{aligned}
$$

where $\Pi_{\mathcal{F}}$ is the projection onto the set $\mathcal{F}$ operator which it can be shown to be given for an arbitrary matrix $\mathbf{A}$ and its projection onto the set $\mathcal{F} \mathbf{A}_{\mathcal{F}}\left(\mathbf{A}_{\mathcal{F}}=\Pi_{\mathcal{F}}\{\mathbf{A}\}\right)$ by [13]

$$
\mathbf{A}_{\mathcal{F}}(m, n)=\left\{\begin{array}{ll}
0, & \mathbf{A}(m, n)=0 \\
\mathbf{A}(m, n) /|\mathbf{A}(m, n)|, & \mathbf{A}(m, n) \neq 0
\end{array},\right.
$$

where $\mathbf{A}_{\mathcal{F}}(m, n)$ and $\mathbf{A}(m, n)$ are the elements at the $m$ th row - $n$th column of matrices $\mathbf{A}_{\mathcal{F}}$ and $\mathbf{A}$ respectively and $|\cdot|$ is the modulus of a complex number.

We move now to the solution of $\left(\mathcal{P}_{4 C}\right)$ which can be shown to admit the following closed form by equating the gradient of (10) with respect to $\mathbf{F}_{B B}$ to zero,

$$
\mathbf{F}_{B B(n)}=\frac{1}{\alpha}\left(\mathbf{F}_{R F(n)}^{H} \mathbf{F}_{R F(n)}\right)^{-1} \mathbf{F}_{R F(n)}^{H}\left(\boldsymbol{\Lambda}_{n-1}+\alpha \mathbf{Z}_{n}\right) .
$$

Finally, Lagrange multiplier $\left(\mathcal{P}_{4 D}\right)$ is updated via the following gradient ascent step

$$
\boldsymbol{\Lambda}_{n}=\boldsymbol{\Lambda}_{n-1}+\alpha\left(\mathbf{Z}_{n}-\mathbf{F}_{R F(n)} \mathbf{F}_{B B(n)}\right) .
$$

Upon convergence of the ADMM sequence, we project the digital pre-coder solution to the set $\mathcal{S}^{\prime}$ defined as, $\mathcal{S}^{\prime}=$ $\left\{\mathbf{A} \in \mathbb{C}^{T_{s} \times N_{s t}} \mid\left\|\mathbf{F}_{R F(\dagger)} \mathbf{A}\right\|_{F}^{2} \leq P_{\max }\left\|\mathbf{H}_{p s} \mathbf{F}_{R F(\dagger)} \mathbf{A}\right\|_{F}^{2} \leq\right.$ $\left.I_{\max }\right\}$ where $\mathbf{F}_{R F(\dagger)}$ is the solution for the analog pre-coder provided by the ADMM sequence and $\mathbf{A}$ is again, an arbitrary matrix. Similarly to (16), it can be shown that for $\mathbf{A} \notin \mathcal{S}^{\prime}$ the projection onto $\mathcal{S}^{\prime}$ is given by [13]

$$
\Pi_{\mathcal{S}^{\prime}}\{\mathbf{A}\}=\mathbf{C}^{-1} \mathbf{A}
$$

where $C=\mathbf{I}_{L_{s}}+\mathbf{F}_{R F(\dagger)}^{H}\left(\gamma_{1} \mathbf{I}_{T_{s}}+\gamma_{2} \mathbf{H}_{p s}^{H} \mathbf{H}_{p s}\right) \mathbf{F}_{R F(\dagger)}$ and the Lagrange Multipliers $\gamma_{1}$ and $\gamma_{2}$ are again set such that the power $\left(P_{\max }\right)$ and interference constraints $\left(I_{\max }\right)$ are met.

Regarding the convergence of the ADMM sequence (11)(14) to an optimal point, the following theorem is given Theorem 1: Let $\left\{\boldsymbol{\Theta}_{n}\right\}=\left\{\mathbf{Z}_{n}, \mathbf{F}_{R F(n)}, \mathbf{F}_{B B(n)}, \boldsymbol{\Lambda}_{n}\right\}$ is a sequence generated by the alternating minimization steps (11)(14). Let us further assume that the multiplier sequence $\left\{\boldsymbol{\Lambda}_{n}\right\}$ is bounded and satisfies $\sum_{n=0}^{\infty}\left\|\boldsymbol{\Lambda}_{n}-\boldsymbol{\Lambda}_{n-1}\right\|_{F}^{2}<\infty$. Then, $\left\{\boldsymbol{\Theta}_{n}\right\}$ converges always to an optimal point of $\left(\mathcal{P}_{4}\right)$.

Proof: The proof can be found in [13].

The implementation aspects of the proposed approach are discussed in [13]. The complete procedure is given for reference in Algorithm 1.

Let us now move to the derivation of the post-coding matrix by expressing the corresponding optimization problem as

$$
\begin{array}{ll}
\left(\mathcal{P}_{7}\right): & \min \mathbb{\mathbf { W } _ { R B } , \mathbf { W } _ { B B }} \mathbb{E}\left\{\left\|\mathbf{x}_{s}-\mathbf{W}_{B B}^{H} \mathbf{W}_{R F}^{H} \mathbf{y}_{s}\right\|_{F}^{2}\right\} \\
& \text { s.t. } \quad \mathbf{W}_{R F} \in \mathcal{F} .
\end{array}
$$

In the absence of hardware limitations that pose the phase only constraints on the receiver, one may find the corresponding optimal digital only post-coder by solving again the unconstrained problem of (5) given that the hybrid pre-coding solutions of Algorithm 1 are applied on the transmitter side. Thus, by denoting with $\mathbf{W}_{D}$ the optimal digital only MMSE post-coder, it is straightforward to see from (6) that,

$$
\begin{aligned}
\mathbf{W}_{D}^{H}= & \left(\mathbf{H}_{s s} \mathbf{F}_{R F} \mathbf{F}_{B B} \mathbf{F}_{B B}^{H} \mathbf{F}_{R F}^{H} \mathbf{H}_{s s}^{H}+\tilde{\mathbf{H}}_{s p} \tilde{\mathbf{H}}_{s p}^{H}+\sigma_{n}^{2} \mathbf{I}_{R_{s}}\right)^{-1} \\
& \times \mathbf{H}_{s s} \mathbf{F}_{R F} \mathbf{F}_{B B} .
\end{aligned}
$$

Returning now to the non-convex problem $\left(\mathcal{P}_{7}\right)$, we first express it in the following equivalent form [3], [13]

$$
\begin{aligned}
& \left(\mathcal{P}_{8}\right): \min _{\mathbf{G}, \mathbf{W}_{R F}, \mathbf{W}_{B B}}\left\|\mathbb{E}\left\{\mathbf{y}_{s} \mathbf{y}_{s}^{H}\right\}^{1 / 2}\left(\mathbf{W}_{D}-\mathbf{G}\right)\right\|_{F}^{2} \\
& +\mathbb{1}_{\mathcal{F}}\left\{\mathbf{W}_{R F}\right\} \\
& \text { s.t. } \mathbf{G}=\mathbf{W}_{R F} \mathbf{W}_{B B},
\end{aligned}
$$



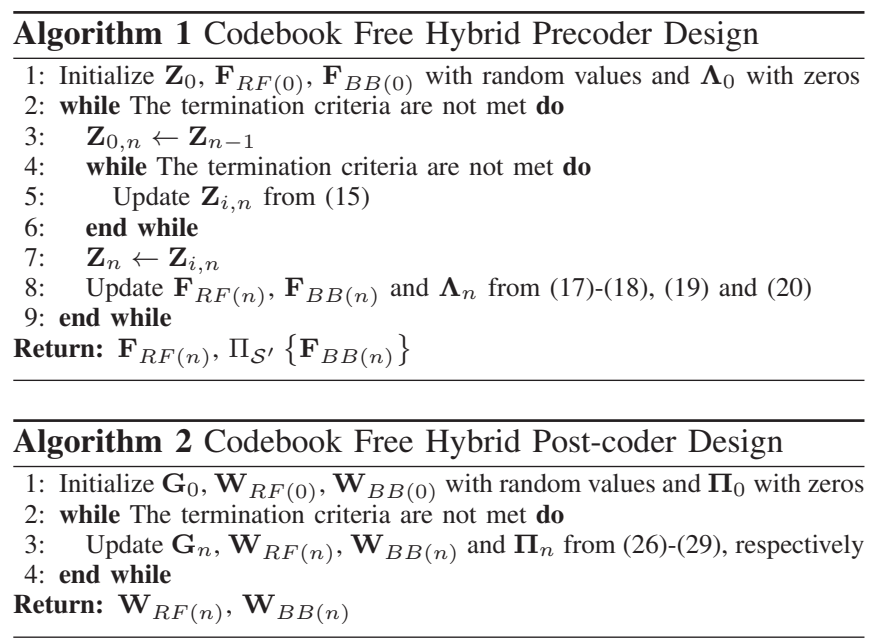

where $\mathbf{G}$ is an auxiliary $R_{s} \times N_{s r}$ matrix variable and the indicator function of set $\mathcal{F}$ is defined as in (9).

The augmented Lagrangian function of $\left(\mathcal{P}_{8}\right)$ is given by

$$
\begin{aligned}
& \mathcal{L}_{R}\left(\mathbf{G}, \mathbf{W}_{R F}, \mathbf{W}_{B B}, \mathbf{\Pi}\right)=\left\|\mathbb{E}\left\{\mathbf{y}_{s} \mathbf{y}_{s}^{H}\right\}^{1 / 2}\left(\mathbf{W}_{D}-\mathbf{G}\right)\right\|_{F}^{2}+ \\
& \mathbb{1}_{\mathcal{F}}\left\{\mathbf{W}_{R F}\right\}+\left\langle\mathbf{\Pi}, \mathbf{G}-\mathbf{W}_{R F} \mathbf{W}_{B B}\right\rangle \\
& +\frac{\beta}{2}\left\|\mathbf{G}-\mathbf{W}_{R F} \mathbf{W}_{B B}\right\|_{F}^{2}
\end{aligned}
$$

where $\Pi$ is the $R_{s} \times N_{s r}$ Lagrange Multiplier matrix and $\beta$ is a scalar penalty parameter.

Following again the ADMM methodology, the solution of matrices $\mathbf{G}, \mathbf{W}_{R F}$ and $\mathbf{W}_{B B}$ is given via an alternating minimization procedure similar to the one of (11)-(14). Due to space limitations we provide directly the solutions.

Thus, matrix $\mathbf{G}_{n}$ is updated via

$$
\begin{aligned}
& \mathbf{G}_{n}=\left(\mathbb{E}\left\{\mathbf{y} \mathbf{y}^{H}\right\}+\beta \mathbf{I}_{R_{s}}\right)^{-1} \times \\
& \left(\mathbb{E}\left\{\mathbf{y} \mathbf{y}^{H}\right\} \mathbf{W}_{D}-\mathbf{\Pi}_{n-1}+\beta \mathbf{W}_{R F(n-1)} \mathbf{W}_{B B(n-1)}\right) .
\end{aligned}
$$

The matrix $\mathbf{F}_{R F(n)}$, is correspondingly given by,

$$
\begin{aligned}
\tilde{\mathbf{W}}_{R F(n)} & =\frac{1}{\beta}\left(\boldsymbol{\Pi}_{n-1}+\beta \mathbf{G}_{n}\right) \mathbf{W}_{B B(n)}^{H}\left[\mathbf{W}_{B B(n)} \mathbf{W}_{B B(n)}^{H}\right]^{-1} \\
\mathbf{W}_{R F(n)} & =\Pi_{\mathcal{F}}\left\{\tilde{\mathbf{W}}_{R F(n)}\right\}
\end{aligned}
$$

Furthermore, matrix $\mathbf{F}_{B B(n)}$ is updated via the following equation

$$
\mathbf{W}_{B B(n)}=\frac{1}{\beta}\left(\mathbf{W}_{R F(n)}^{H} \mathbf{W}_{R F(n)}\right)^{-1} \mathbf{W}_{R F(n)}^{H}\left(\mathbf{\Pi}_{n-1}+\beta \mathbf{G}_{n}\right) .
$$

Finally, $\boldsymbol{\Pi}$ is updated via a gradient ascend step, that is

$$
\boldsymbol{\Pi}_{n}=\boldsymbol{\Pi}_{n-1}+\beta\left(\mathbf{G}_{n}-\mathbf{W}_{R F(n)} \mathbf{W}_{B B(n)}\right) .
$$

Algorithm 2 summarizes the procedure.

\section{Codebook Based Hybrid Transceiver Design}

Let us assume that the analog precoders the SU transceiver ends are determined from a codebook. Here, we assume a finite dictionary derived from the uniform quantization of the azimuth angles over the interval $[0,2 \pi]$. Thus, the columns of matrices $\mathbf{F}_{R F}$ and $\mathbf{W}_{R F}$ must satisfy $\mathbf{F}_{R F}^{(i)}\left(\mathbf{W}_{R F}^{(i)}\right) \in$
$\mathcal{C}_{\phi}=\left\{\mathbf{a}\left(\phi_{1}\right), \ldots, \mathbf{a}\left(\phi_{2^{N_{\phi}}}\right)\right\}$, respectively, where $N_{\phi}$ are the quantization bits for the azimuth angles.

Let us start with the solution for the precoding matrix $\mathbf{F}_{R F}$. The approach of [3] is followed where the hybrid pre-coder is designed such that the Frobenious norm of its difference to the optimal digital only solution is minimized. Thus, the following optimization problem is defined,

$$
\begin{array}{ll}
\left(\mathcal{P}_{9}\right): & \min \left\|\mathbf{F}_{D}-\mathbf{F}_{R F} \mathbf{F}_{B B}\right\|_{F}^{2} \\
& \text { s.t. } \mathbf{F}_{R F} \mathbf{F}_{B B} \in \mathcal{S} \& \mathbf{F}_{R F} \in \mathcal{F}_{R F}
\end{array}
$$

where the optimal digital only solution $\mathbf{F}_{D}$ is computed by solving $\left(\mathcal{P}_{2}\right)$ in Section III and $\mathcal{F}_{R F}$ is the feasible set for the analog precoder. $\left(\mathcal{P}_{9}\right)$ can be cast as a sparse representation problem over the dictionary $\mathcal{C}_{\phi}$, that is

$$
\begin{aligned}
\left(\mathcal{P}_{10}\right): & \min _{\tilde{\mathbf{F}}_{B B}}\left\|\mathbf{F}_{D}-\mathbf{\Phi} \tilde{\mathbf{F}}_{B B}\right\|_{F}^{2} \\
& \text { s.t. } \boldsymbol{\Phi} \tilde{\mathbf{F}}_{B B} \in \mathcal{S} \&\left\|\tilde{\mathbf{F}}_{B B} \tilde{\mathbf{F}}_{B B}^{H}\right\|_{0}=N_{t},
\end{aligned}
$$

where $\|\cdot\|_{0}$ is the $l_{0}$ norm, $\tilde{\mathbf{F}}_{B B}$ is a $2^{N_{\phi}} \times N_{s t}$ matrix of at most $N_{s t}$ no zero rows and $\boldsymbol{\Phi}=\left[\mathbf{a}\left(\phi_{1}\right), \ldots, \mathbf{a}\left(\phi_{2^{N_{\phi}}}\right)\right]$. The solution to $\left(\mathcal{P}_{10}\right)$ can be derived via the Orthogonal Matching Pursuit (OMP) concept from the relevant sparse representation/reconstruction literature [15], as it is presented in [3]. The solution is given by Algorithm 1 of [3], with the difference that the result in line 7 of this algorithm is projected onto the set $\mathcal{S}^{\prime}$ in order to satisfy the power and interference constraints in $\left(\mathcal{P}_{10}\right)$

Let us move now to the design of the proposed codebook based hybrid solution for the SU receiver. It is easy to see that we can cast directly problem $\left(\mathcal{P}_{7}\right)$ to the codebook based case via the following sparse representation problem

$$
\begin{aligned}
\left(\mathcal{P}_{11}\right): & \min _{\tilde{\mathbf{W}}_{B B}}\left\|\mathbb{E}\left\{\mathbf{y}_{s} \mathbf{y}_{s}^{H}\right\}^{1 / 2}\left(\mathbf{W}_{D}-\mathbf{\Phi} \tilde{\mathbf{W}}_{B B}\right)\right\|_{F}^{2} \\
& \text { s.t. }\left\|\operatorname{diag}\left(\tilde{\mathbf{W}}_{B B} \tilde{\mathbf{W}}_{B B}^{H}\right)\right\|_{0}=N_{s r},
\end{aligned}
$$

where $\tilde{\mathbf{W}}_{B B}$ is a $2^{N_{\phi}+{ }_{\theta}} \times N_{s r}$ matrix of at most $N_{s r}$. The solution to $\left(\mathcal{P}_{11}\right)$ is given again via an OMP-based solution which can be found in [3] with the difference that values $\mathbb{E}\left\{\mathbf{y}_{s} \mathbf{y}_{s}^{H}\right\}$ and $\mathbf{W}_{D}$ have to be replaced with the corresponding ones of the present paper.

\section{Numerical RESUlts AND Discussion}

In this section, numerical results are presented for evaluating the performance of the proposed hybrid approaches An environment of $N_{p}=15$ propagation paths is assumed for all the involved links (SU-to SU, the SU-to-PU and PUto-SU). The codebook $\boldsymbol{\Phi}$ is designed as described in [3] for $N_{\Phi}=8$ bits. The performance of the proposed approaches is examined in terms of the achieved mean spectral efficiency versus the Signal-to-Noise-Ratio (SNR) over 100 channel realizations. We now refer to the parameter tuning of the proposed Algorithms. The following numbers are the same for all the experiments presented in this paper. For Algorithm 1, the parameters are set as $\mu=10^{-3}$ and $\alpha=10$. For Algorithm 2 , we set $\beta=1$. Termination criteria for both Algorithms 1 and 2 can be found in [13].

In Fig. 2.a, the performance of the different hybrid techniques is examined for a SU $64 \times 16$ MIMO system with $N_{s t}=N_{s r}=\{2,4,6\}$ RF chains. The performance of the 


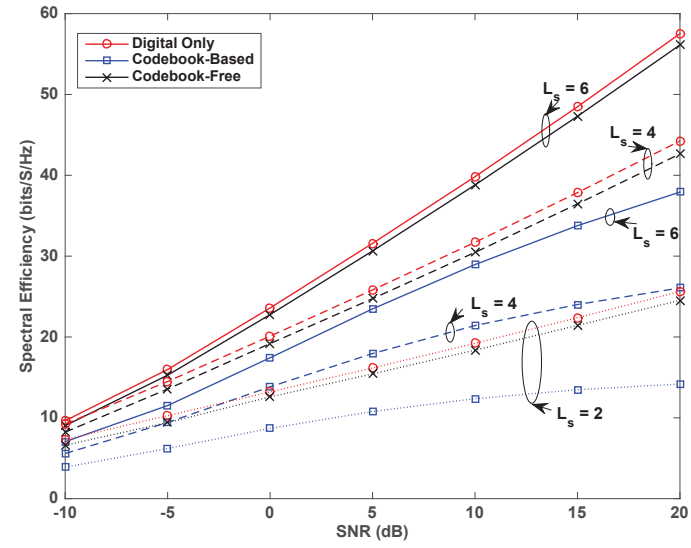

(a)

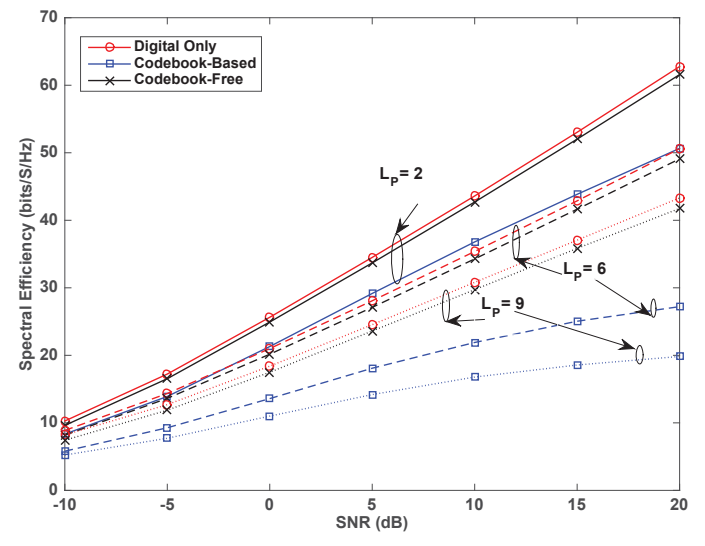

(b)

Fig. 2. Spectral Efficiency of the Hybrid Transceivers vs SNR for a $64 \times 16$ MIMO system for a) Different number of RF Chains, $N_{s t}=N_{s r}=L_{s}=\{2,4,6\}$ and b) Different PU signal's Rank, $L_{p}=\{2,6,9\}$.

digital only solution is also plotted for $L_{s}=\{2,4,6\}$ for a fair comparison, since the hybrid approaches can support transmission of maximum $\min \left\{N_{s t}, N_{s r}\right\}$ streams. The maximum transmission power and interference constraints are set to $P_{\max }=1$ and $I_{\max }=1$, respectively. The PU is assumed to transmit a signal of rank $L_{p}=4$. As its shown, the codebook free hybrid approach achieves close performance to the one of the digital only solution for all the examined cases. Moreover, the performance of the codebook based technique is severely inferior to the one of the codebook based solution, especially for high SNR values. This is due to the codebook use that restricts the solution set of the analog counterpart, as it was also observed in [4], [5].

In Fig. 2.b, we examine the impact of the PU signal's rank on the performance of the proposed techniques. The number of RF chains is fixed to $N_{s t}=N_{s r}=6$ and $I_{\max }=1$. We consider PU signals of rank $L_{p}=\{2,6,9\}$ respectively. The rest of the parameters are the same with the one of the experiment of Fig. 2.a. As it was expected, the performance of all the techniques is degraded as the rank of PU signal's increases. This can be attributed to the fact that more of the available degrees of freedom are occupied by the PU, as its signal's rank increases and thus, the SU has to reduce further its transmitted power in order to satisfy the interference temperature constraint. The proposed codebook free hybrid approach achieve once again close performance to the one of the digital only optimal solution, independently of the PU signal's rank. On the contrary, the performance of the codebook based approach is degraded with an increase on the PU signal's of rank.

Future works that are currently under development include the extension of the proposed approach to partially connected hybrid solutions and frequency selective channels.

\section{ACKNOWLEDGMENT}

This work is partially supported by the FNR, Luxembourg under the projects SeMIGod, and SATSENT as well as European project $\mathrm{H} 2020$ SANSA.

\section{REFERENCES}

[1] T. Bai and R. W. Heath, "Coverage and rate analysis for millimeter-wave cellular networks," IEEE Transactions on Wireless Communications, vol. 14, no. 2, pp. 1100-1114, Feb 2015.

[2] T. S. Rappaport, G. R. MacCartney, M. K. Samimi, and S. Sun, "Wideband millimeter-wave propagation measurements and channel models for future wireless communication system design," IEEE Transactions on Communications, vol. 63, no. 9, pp. 3029-3056, Sept 2015.

[3] O. E. Ayach, S. Rajagopal, S. Abu-Surra, Z. Pi, and R. W. Heath, "Spatially sparse precoding in millimeter wave MIMO systems," IEEE Transactions on Wireless Communications, vol. 13, no. 3, pp. 14991513, March 2014.

[4] X. Yu, J. C. Shen, J. Zhang, and K. B. Letaief, "Alternating minimization algorithms for hybrid precoding in millimeter wave MIMO systems," IEEE Journal of Selected Topics in Signal Processing, vol. 10, no. 3, pp. 485-500, April 2016.

[5] F. Sohrabi and W. Yu, "Hybrid digital and analog beamforming design for large-scale antenna arrays," IEEE Journal of Selected Topics in Signal Processing, vol. 10, no. 3, pp. 501-513, April 2016.

[6] I.-R. R. Regulations, "Frequency allocations," Volume I, Chapter II, Article 5, 2012.

[7] A. Goldsmith, S. A. Jafar, I. Maric, and S. Srinivasa, "Breaking spectrum gridlock with cognitive radios: An information theoretic perspective," Proceedings of the IEEE, vol. 97, no. 5, pp. 894-914, May 2009.

[8] D. P. Palomar and M. Chiang, "A tutorial on decomposition methods for network utility maximization," IEEE Journal on Selected Areas in Communications, vol. 24, no. 8, pp. 1439-1451, Aug 2006.

[9] F. D. Neeser and J. L. Massey, "Proper complex random processes with applications to information theory," IEEE Transactions on Information Theory, vol. 39, no. 4, pp. 1293-1302, Jul 1993.

[10] S. Boyd and L. Vandenberghe, "Convex optimization," 2004.

[11] T. Kailath, A. H. Sayed, and B. Hassibi, Linear estimation. Prentice Hall Upper Saddle River, NJ, 2000, vol. 1.

[12] S. Boyd, N. Parikh, E. Chu, B. Peleato, and J. Eckstein, "Distributed optimization and statistical learning via the alternating direction method of multipliers," Foundations and Trends $囚$ in Machine Learning, vol. 3, no. 1, pp. 1-122, 2011.

[13] C. G. Tsinos and S. Maleki and S. Chatzinotas and B. Ottersten, "Hybrid analog-digital transceiver designs for cognitive large-scale antenna array systems," IEEE Trans. on Signal Processing, 2016, Submitted.

[14] D. P. Bertsekas, "Nonlinear programming," 1999.

[15] J. A. Tropp and A. C. Gilbert, "Signal recovery from random measurements via orthogonal matching pursuit," IEEE Trans. on Information Theory, vol. 53, no. 12, pp. 4655-4666, Dec 2007. 